\title{
Capacitación de médicos residentes en el reconocimiento y tratamiento de un caso de anafilaxia en pediatría con modelos de simulación
}

\section{Training of resident physicians in the recognition and treatment of an anaphylaxis case in pediatrics with simulation models}

\author{
Dr. Diego Enríquez, ${ }^{a}$ Dra. María J. Lamborizio, ${ }^{b}$ Dra. Lorena Firenze, ${ }^{b}$ \\ Dra. María de la P. Jaureguizar, ${ }^{b}$ Dr. Estanislao Díaz Pumaráb y Dr. Edgardo Szyld
}

\section{RESUMEN}

Objetivo. Evaluar el desempeño logrado por médicos residentesenel diagnósticoy tratamiento de un caso simulado de anafilaxia luego de 6 meses dehaber recibido capacitación con técnicas de simulación.

Material y método. Un grupo de residentes de Pediatría fueron capacitados a través de técnicas de simulación en diferentes emergencias pediátricas (primera etapa) y luego clasificados según su grado de desempeño en 3 subgrupos. Se seleccionaron, posteriormente, 4 médicos al azar de cada subgrupo para ser reevaluados luego de 6 meses (segunda etapa).

Resultados. Participaron 72 médicos de la primera etapa, $56(77,8 \%)$ resolvieron el caso correctamente. A los 6 meses, los $12(100 \%)$ médicos evaluados trataron en forma adecuada el nuevo caso de anafilaxia.

a. Centro de Simulación Médica de Roemmers (SIMMER), Olivos, Pcia. de Buenos Aires.

b. Hospital de Niños Ricardo Gutiérrez, Ciudad Autónoma de Buenos Aires.

c. Oklahoma

University

Health Science

Center (OUHSC),

Oklahoma City;

Icahn School of

Medicine, Mt.

Sinai, Nueva York,

Estados Unidos de América.

Correspondencia:

Dr. Diego Enríquez: dsenriquez2000@yahoo. com.ar

Financiamiento:

Ninguno.

Conflicto de intereses: Ninguno que declarar.

Recibido: 14-12-2016

Aceptado: 29-3-2017 de simulación permitió corregir u optimizar el tratamiento de casos simulados de anafilaxia en médicos residentes evaluados luego de 6 meses de la capacitación inicial.

Palabras clave: anafilaxia, simulación, residencia médica.

\section{ABSTRACT}

Objective: Toevaluate the performance of resident physicians in diagnosing and treating a case of anaphylaxis, six months after participating in simulation training exercises.

Methods: Initially, a group of pediatric residents were trained using simulation techniques in the management of critical pediatric cases. Based on their performance in this exercise, participants were assigned to one of 3 groups. At six months post-training, 4 residents were randomly chosen from each group to be re-tested, using the same performance measure as previously used.

Results: During theinitial training session, 56 of 72 participants $(78 \%)$ correctly identified and treated the case. Six months after the initial training, all $12(100 \%)$ resident physicians who were re-tested successfully diagnosed and treated the simulated anaphylaxis case.

\section{Colaboradores:}

Conclusions: The training through simulation techniques allowed correction or optimization of the treatment of simulated anaphylaxis cases in resident physicians evaluated after 6 months of the initial training.

Key words: anaphylaxis, simulation, medical residence.

http:/ / dx.doi.org/10.5546/ aap.2017.399

Cómo citar: Enríquez D, Lamborizio MJ, Firenze L, et al. Capacitación de médicos residentes en el reconocimiento y tratamiento de un caso de anafilaxia en pediatría con modelos de simulación. Arch Argent

\section{INTRODUCCIÓN}

En la última década, se ha Pediatr 2017;115(4):399-403. observado un aumento de la frecuencia de cuadros de naturaleza alérgica en los niños, en particular, de origen alimentario. De igual modo, se han incrementado las consultas a los servicios de emergencia, debido a las manifestaciones agudas y progresivas de la enfermedad alérgica. ${ }^{1-3}$

Las causas de anafilaxia, al menos, en pacientes pediátricos, son, principalmente, producto de la ingestión de fármacos o alimentos y por picadura de insectos. Es por ello por lo que su pesquisa cobra particular importancia, más allá de que el tratamiento inmediato sea idéntico para todos los casos. ${ }^{4,5}$

El tratamiento recomendado en la actualidad para los cuadros de anafilaxia en la infancia consiste,
Dr. Nicolás A. Falk, Dr. Agustín Iglesias y Dra. Josefina Fernández Díaz (instructores de residentes del Hospital de Niños Ricardo Gutiérrez). 
principalmente, en la administración parenteral precoz de adrenalina. La demora en su administración (por lo general, debida a un diagnóstico inicial erróneo) se asocia con una evolución adversa del enfermo y un aumento del riesgo de muerte..$^{6-8}$

La anafilaxia es un cuadro clínico asociado a un escaso reconocimiento por parte de los profesionales y a una aplicación infrecuente de las guías de tratamiento, aun en los Estados Unidos (EE. UU.). Debido a esto, la mayoría de los individuos en riesgo de sufrir un evento anafiláctico no recibe el tratamiento recomendado. ${ }^{1,9}$ En función de la relevancia de este tipo de patologías y la necesidad de diagnóstico y tratamientos inmediatos, es conveniente evaluar las competencias relacionadas dentro de los sistemas de formación de médicos residentes.

La metodología de enseñanza médica basada en la simulación de casos se utiliza cada vez con mayor frecuencia en nuestros días, dado que permite aumentar la destreza manual y facilita la fijación de la información necesaria para actuar. ${ }^{10}$ En pediatría, se la utiliza para evaluar diferentes escenarios clínicos, como la aplicación de las técnicas de reanimación, el manejo de la vía aérea, la resolución de situaciones de crisis o para el aprendizaje de diferentes técnicas de procedimientos médicos. ${ }^{11}$

\section{OBJETIVOS}

El objetivo principal fue evaluar el desempeño logrado por un grupo de médicos residentes de Pediatría en el diagnóstico y tratamiento de la anafilaxia luego de 6 meses de haber recibido una capacitación a través de las técnicas de simulación clínica.

El objetivo secundario fue describir el desempeño de los médicos residentes de Pediatría en las diferentes etapas del caso de anafilaxia simulado (tiempo al momento del diagnóstico, tiempos hasta la indicación y administración efectiva de adrenalina y vía de administración elegida) en esa primera capacitación.

\section{POBLACIÓN Y MÉTODOS}

Este estudio incluyó a médicos residentes de Pediatría del Hospital de Niños Ricardo Gutiérrez de la Ciudad Autónoma de Buenos Aires. Se invitó, a través del Comité de Docencia e Investigación del Hospital, a todos los residentes (de primero a tercer año) a participar en la primera etapa del estudio.

Dentro del marco de la primera etapa de capacitación de dos jornadas de 4 horas de duración cada una, se capacitó a través del conjunto de técnicas de simulación ${ }^{12,13}$ en el manejo de pacientes críticos y reanimación cardiopulmonar (RCP). Durante estas, se incluyó, además, el caso de un niño de 2 años que fue traído a la consulta en la sala de emergencias con un cuadro de anafilaxia.

Este caso fue previamente validado a través de un proyecto piloto ante un grupo de siete pediatras especialistas y con trabajo activo en sectores de emergencias para establecer tanto la fidelidad del escenario como los estándares de comportamiento esperados (guías de manejo clínico habituales en nuestro medio). ${ }^{14}$ Participaron en el caso clínico simulado en forma individual y todos llegaron al diagnóstico correcto.

\section{Primera etapa de capacitación}

La primera etapa se realizó durante diferentes días con médicos residentes divididos por grupos. La duración fue de 8 horas en total divididas en 2 días. El primer día constaba de entrenamiento de habilidades prácticas en RCP con simuladores de baja fidelidad (compresiones torácicas), ventilación con bolsa y máscara, utilización del desfibrilador externo automático, colocación de accesos intraóseos e intravasculares e intubación endotraqueal. El segundo día constaba de 4 casos simulados de alta fidelidad de urgencias pediátricas; algunos incluían la necesidad de realizar maniobras de RCP.

Durante esta etapa con casos clínicos simulados de pacientes pediátricos críticos, se cumplieron con todas las fases de las jornadas de simulación: información previa del curso teórico de RCP pediátrico, desarrollo del caso clínico y "debriefing", anglicismo que se refería a las reuniones de evaluación y devolución teóricas, posteriores a las acciones realizadas durante las simulaciones. Durante la fase de "debriefing", se detalló la actuación observada y, bajo técnicas específicas, se indujo a los participantes a la determinación de los procedimientos que se habían ejecutado en forma correcta y cuáles debieran haberse modificado. De esta forma, finalizó cada caso con la reflexión por parte de los participantes acerca de los distintos temas.

Al finalizar cada jornada de la primera etapa, se les solicitó a los participantes la firma de un acuerdo de confidencialidad y del consentimiento para ser invitados a regresar eventualmente a los 6 meses de realizada la primera etapa. Todos firmaron dicho consentimiento. De los tres grupos surgidos de la clasificación por el desempeño de la primera etapa, se eligieron cuatro residentes por grupo en forma aleatoria, utilizando una tabla generada por computadora. El pedido de consentimiento informado fue solicitado antes de la aleatorización para que ningún participante 
supiera el porqué de la segunda convocatoria al centro de simulación.

Posteriormente, se realizó una evaluación del desempeño en el caso de anafilaxia. Se clasificó a los médicos residentes en tres grupos: grupo 1, los que no habían llegado nunca al diagnóstico; grupo 2, los que habían llegado al diagnóstico y errado el tratamiento; y grupo 3, los que habían completado satisfactoriamente la evaluación cumpliendo con las guías correspondientes.

Para la segunda etapa, se tomó una muestra de 4 residentes de cada grupo mencionado y se los invitó a participar de una nueva jornada de capacitación a los 6 meses de la primera. Los participantes no conocían el motivo de su elección para esta fase del estudio y solo se les informó que, en esa oportunidad, debían resolver nuevamente, al menos, tres casos de pacientes críticos. Se les presentó otro caso de anafilaxia para su diagnóstico y tratamiento cambiando la edad del paciente simulado (el de 2 años por uno de 6 ) y las características del escenario (el primero, en sala de emergencias, y el segundo, en un domicilio particular), pero con igual presentación clínica y parámetros del caso de la primera etapa.

Las variables registradas para el caso del paciente simulado con anafilaxia en ambas etapas fueron los tiempos hasta la indicación oral y administración efectiva de adrenalina, vía de administración y la indicación de otros tratamientos coadyuvantes.

La simulación se realizó sobre el modelo SIM one year old $\left(\right.$ Gaumard $\left.^{\circledR}\right)$ para el caso de la primera etapa y el SIM five year old (Gaumard ${ }^{\circledR}$ ) para la segunda. Para la recreación durante los casos clínicos de las manifestaciones dermatológicas de la anafilaxia con maculopápulas y edema de labios, se utilizó un set de maquillaje.

Se cronometró desde el inicio del caso hasta la administración intramuscular de adrenalina en los casos donde se la indicara.

La variable de resultado primario fue la proporción de médicos residentes de la segunda etapa que completaron la prueba dentro del rango definido previamente como satisfactorio.

Se registraron, además, las siguientes variables de resultado secundario:

- Tiempo de administración de la adrenalina (en el que se entendía que el alumno llegaba al diagnóstico correcto de anafilaxia), número de dosis correctas y de técnicas aplicadas.

- Proporción de participantes que cumplieron con todos los pasos y tiempos esperados en la segunda etapa, comparados con su desempeño en la primera etapa.

Las variables descritas fueron obtenidas de la grabación en video o extraídas del registro automático del monitor a través de la interfase conectada a una computadora (tableta).

\section{Manejo de datos}

Todos los parámetros incluidos en las variables de resultado fueron volcados a una base de datos diseñada para tal fin (planilla Microsoft ${ }^{\circledR}$ Excel $^{\circledR}$ 2013).

\section{Tamaño muestral y análisis estadístico}

El tamaño muestral del presente estudio en su segunda etapa fue de 12 médicos, número elegido por conveniencia, dado que era el primer estudio que se realizaba con esta población y era difícil predecir la homogeneidad de la muestra.

Las variables continuas se describieron como medias y desvío estándar. Las proporciones se describieron como porcentaje e intervalo de confianza (IC) del 95\%. Para comparar las proporciones, se utilizó el test de Student. Se consideró significativo un valor de $\mathrm{p}$ menor de 0,05 .

Los datos del estudio se manejaron en forma anónima asignando un código numérico a cada participante de modo tal que no pudieran ser identificados. La base de datos se encuentra registrada en la Dirección Nacional de Protección de Datos Personales del Ministerio de Justicia, Seguridad y Derechos Humanos.

El protocolo fue aprobado por el Comité de Docencia e Investigación del centro participante.

\section{RESULTADOS}

Participaron en el estudio, durante la primera etapa, 72 médicos residentes en 24 jornadas de 4 horas cada una.

Su desempeño se describe en la Tabla 1 según el año de residencia.

En la Tabla 2, se presentan los tiempos requeridos para llegar al diagnóstico correcto, indicar la adrenalina y su aplicación efectiva.

A los 6 meses de la primera etapa, concurrieron los 12 médicos seleccionados. En esta segunda etapa, todos los médicos resolvieron de forma adecuada el nuevo caso de anafilaxia.

En la Tabla 3, se presentan los tiempos utilizados por los médicos con desempeño óptimo en la primera etapa y los empleados en la segunda.

\section{DISCUSIÓN}

A los seis meses de la capacitación inicial, los médicos residentes evaluados mostraron una franca mejora en su desempeño ante un caso de anafilaxia simulado.

Las técnicas de simulación de alta fidelidad 
permiten recrear el escenario frente a la emergencia con mucho realismo, lo cual facilita el aprendizaje, complementado con el "debriefing", que genera un alto grado de retención del conocimiento incorporado. ${ }^{15,16}$

En un estudio realizado por Mills y col., se comparó el desempeño al año de capacitación en reanimación pediátrica con un grupo control histórico y, si bien observaron escasa diferencia en general, se produjo un aumento en las intubaciones realizadas por los residentes, de manera secundaria a un aumento en la autoconfianza. ${ }^{17}$

En otro estudio recientemente publicado, Sidhu y col. evaluaron en forma retrospectiva el desempeño en el manejo clínico de los casos de anafilaxia en un hospital pediátrico de los EE. UU. durante un período de 8 años. Compararon, además, los casos manejados antes y después de la aparición de las guías del Instituto de Alergia y Enfermedades Infecciosas de EE. UU. (National Institute of Allergy and Infectious Diseases, NIAID) $\mathrm{y}$, si bien describieron una marcada mejora en el desempeño luego de la aparición de las guías, todavía solo el $46 \%$ de los pacientes había recibido adrenalina intramuscular, lo que destacaba la necesidad de capacitación en este tema. ${ }^{9}$

Shah y col., por su parte, publicaron un análisis retrospectivo acerca del impacto de la capacitación simulada sobre el manejo de pacientes que presentaban convulsiones. Encontraron una diferencia significativa en el desempeño entre aquellos miembros del personal paramédico que habían recibido capacitación con simulación y aquellos que no. ${ }^{18}$

En nuestro estudio, el impacto de la capacitación realizada mostró mejoría no solo en aquellos médicos que no habían resuelto el caso favorablemente en la primera etapa, sino en

TABla 1. Clasificación en grupos según desempeño y año de residencia

\begin{tabular}{lcccccc}
\hline Desempeño & $\mathbf{N}$ & $\%$ & \multicolumn{3}{c}{ Año de residencia } \\
\cline { 4 - 7 } & & & $\mathbf{1}^{\text {ro }} \mathbf{y ~ 2 ~}^{\text {do }}$ & $\%$ & \multicolumn{1}{c}{$3^{\text {ro }}$} & $\%$ \\
\hline Grupo 1 & 7 & 9,7 & 7 & 20,0 & 0 & 0 \\
Grupo 2 & 9 & 12,5 & 5 & 14,3 & 4 & 10,8 \\
Grupo 3 & 56 & 77,8 & 23 & 65,7 & 33 & 89,02 \\
Total & 72 & 100,0 & 35 & 100 & 37 & 100 \\
\hline
\end{tabular}

Grupo 1: participantes que cometieron un error diagnóstico.

Grupo 2: participantes con error en el tratamiento.

Grupo 3: participantes con diagnóstico y tratamientos realizados correctamente.

Tabla 2. Primera etapa. Tiempos requeridos para llegar al diagnóstico correcto, indicar la adrenalina y su aplicación

\begin{tabular}{|c|c|c|c|c|c|c|c|c|}
\hline \multicolumn{3}{|c|}{$\begin{array}{l}\text { Segundos hasta el } \\
\text { diagnóstico correcto }\end{array}$} & \multicolumn{3}{|c|}{$\begin{array}{l}\text { Segundos hasta la indicación } \\
\text { de administrar adrenalina }\end{array}$} & \multicolumn{3}{|c|}{$\begin{array}{c}\text { Segundos hasta la } \\
\text { administración efectiva }\end{array}$} \\
\hline \multicolumn{2}{|c|}{$\begin{array}{c}\text { Participantes } \\
\quad \mathrm{N}=72 \\
\end{array}$} & \multirow{2}{*}{$\begin{array}{c}\begin{array}{c}\text { Grupo } \\
\text { de expertos* }\end{array} \\
33\end{array}$} & \multicolumn{2}{|c|}{$\begin{array}{c}\text { Participantes } \\
\quad \mathrm{N}=72 \\
\end{array}$} & \multirow{2}{*}{$\begin{array}{c}\begin{array}{c}\text { Grupo } \\
\text { de expertos* }\end{array} \\
50\end{array}$} & \multicolumn{2}{|c|}{$\begin{array}{c}\text { Participantes } \\
\quad \mathrm{N}=72 \\
\end{array}$} & \multirow{2}{*}{$\begin{array}{c}\begin{array}{c}\text { Grupo } \\
\text { de expertos }\end{array} \\
89\end{array}$} \\
\hline Media & 55 & & Media & 89 & & Media & 124 & \\
\hline Mediana & 45 & 33 & Mediana & 60 & 45 & Mediana & 90 & 88 \\
\hline $\mathrm{DE}$ & 36,8 & 7,8 & $\mathrm{DE}$ & 63,1 & 10,7 & $\mathrm{DE}$ & 86,7 & 18,7 \\
\hline Mínimo & 25 & 20 & Mínimo & 30 & 38 & Mínimo & 1,5 & 66 \\
\hline Máximo & 195 & 45 & Máximo & 243 & 65 & Máximo & 300 & 122 \\
\hline IC $(95,0 \%)$ & 9,9 & 7,2 & IC $(95,0 \%)$ & 16,9 & 9,9 & IC $(95,0 \%)$ & 23,2 & 17,3 \\
\hline
\end{tabular}

DE: desviación estándar. IC: intervalo de confianza.

${ }^{*}$ Especialistas en emergencias pediátricas que validaron el caso clínico de anafilaxia. $\mathrm{N}=7$.

TABLA 3. Tabla comparativa entre los participantes que realizaron todo el caso correctamente en lo referente a diagnóstico y tratamientos adecuados en las dos etapas

\begin{tabular}{|c|c|c|c|}
\hline & $\begin{array}{c}\text { Primera } \\
\text { etapa (N: } 72)\end{array}$ & $\begin{array}{c}\text { Segunda } \\
\text { etapa (N: } 12)\end{array}$ & $\begin{array}{l}\text { Diferencia } \\
\text { (segundos) }\end{array}$ \\
\hline Segundos hasta el diagnóstico correcto. Media (máx.-min.) & $55(25-195)$ & $45(22-108)$ & 10 \\
\hline Segundos hasta la indicación de administrar adrenalina. Media (máx.-min.) & $89(30-243)$ & $57(30-140)$ & 32 \\
\hline Segundos hasta la administración efectiva. Media (máx.-min.) & $124(90-300)$ & $89(41-210)$ & 35 \\
\hline
\end{tabular}


aquellos que lo habían hecho. Esto podría estar relacionado con el impacto del refrescamiento. Nuevas exposiciones breves han demostrado mejorar el desempeño profesional en otras emergencias afines, como ser el programa de soporte vital básico (basic life support; BLS, por sus siglas en inglés) o la RCP pediátrica. ${ }^{19,20}$

Mielder y col. propusieron un taller intensivo para capacitar estudiantes de Medicina en el manejo de la anafilaxia con una duración de 26 horas docentes. ${ }^{21}$ Recientemente, describieron los resultados del desempeño de los alumnos capacitados en 5 de dichos cursos, que lograron también un diagnóstico apropiado en el 100\% de los casos, pero con un menor desempeño en los casos más graves. ${ }^{22}$

Couto y col., a su vez, compararon dos estrategias de capacitación para emergencias pediátricas (un caso de anafilaxia y otro de taquicardia supraventricular) y no encontraron diferencias en las evaluaciones entre los alumnos capacitados con discusión de casos de modo tradicional y los que habían participado de la simulación. ${ }^{23}$ En cambio, nuestro estudio evaluó el desempeño de los alumnos en tiempo real frente a un escenario simulado, lo que generó una evaluación más completa.

Dentro de las limitaciones del presente estudio, se pueden reconocer las vinculadas con la simulación, ya que se evaluó el desempeño sobre casos simulados y no con pacientes reales. De todos modos, cuando se realiza en un escenario de alta fidelidad, permite reproducir en forma bastante fidedigna lo que sucedería en la vida real.

Teniendo en cuenta que los médicos participantes siguieron expuestos a un programa de capacitación en servicio, es imposible atribuir los resultados de la mejoría exclusivamente a la capacitación recibida. Sin embargo, por un lado, se observó un desempeño subóptimo también en algunos residentes de tercer año $y$, por otro, todos los médicos expuestos a la capacitación mejoraron su desempeño en algún grado.

\section{CONCLUSIÓN}

La capacitación a través de técnicas de simulación permitió corregir u optimizar el tratamiento de casos de anafilaxia simulados en un grupo de médicos pediatras en formación evaluados luego de 6 meses de su capacitación inicial.

\section{REFERENCIAS}

1. Sclar DA, Lieberman PL. Anaphylaxis: underdiagnosed, underreported, and undertreated. Am JMed 2014;127(Suppl 1):S1-5.

2. AustenF.Diseases of immune-mediated injury.In Petersdorf
G, Adams R. Braunwald E,et al (eds.). Harrison's Principles of internal Medicine. 10 th ed. New York: McGraw-Hill; 1993: 372-7.

3. Mora Santiago MR, Muros Fuentes B, Fernández-Ovies JM, et al. Tratamiento del shock anafilactico. A propósito de un caso clínico. Farm Hosp 1999;23(2):131-3.

4. Ortega Casanueva C. Alergia a la Picadura de Insectos. Pediatr Integral 2013;17(9):628-36.

5. Muñoz MG. Alergia a medicamentos. Conceptos básicos y actitud a seguir por el pediatra. Protocolos en diagnóstico de terapéutica pediátrica 2013;1:1-24.

6. Grabenstein J. Anaphylaxis: Epinephrine \& Emergency Responses. Hosp Pharm 1997;32:1377-89.

7. García-Sevilla J, Meana JJ. Transmisión catecolaminérgica. Fármacos agonistas catecolaminérgicos. Farmacología humana 1997;5:295-319.

8. Sampson HA, Muñoz-Furlong A, Campbell RL, et al. Second symposium on the definition and management of anaphylaxis: summary report--Second National Institute of Allergy and Infectious Disease/Food Allergy and Anaphylaxis Network symposium. J Allergy Clin Immunol 2006;117(2):391-7.

9. Sidhu N, Jones S, Perry T, et al. Evaluation of Anaphylaxis Management in a Pediatric Emergency Department. Pediatr Emerg Care 2016;32(8):508-13.

10. Mileder LP, Urlesberger B, Szyld EG, et al.Simulation-based neonatal and infant resuscitation teaching: a systematic review of randomized controlled trials. Klin Padiatr 2014;226(5):259-67.

11. O'Leary FM, Hokin B, Enright $\mathrm{K}$ et al. Treatment of a simulated child with anaphylaxis: an in situ two-arm study. I Paediatr Child Health 2013;49(7):541-7.

12. Brett-Fleegler MB, Vinci RJ, Weiner DL, et al. A simulatorbased tool that assesses pediatric resident resuscitation competency. Pediatrics 2008;121(3):e597-603.

13. Palés ArgullósJL, GomarSancho C. Elusodelas simulaciones eneducación médica. Education in the Knowledge Society (EKS) 2010;11(2):147-70.

14. Rufauch D, Santos S. Reanimación Cardiopulmonar Pediátrica Manual del curso ERA. Buenos Aires: Sociedad Argentina de Pediatría; 2012.

15. OjhaR, Liu A, Rai D, etal. Review of Simulation in Pediatrics: The Evolution of a Revolution. Front Pediatr 2015;3:106.

16. Mills DM, Williams DC, Dobson JV. Simulation training as a mechanism for procedural and resuscitation education for pediatric residents: a systematic review. Hosp Pediatr 2013;3(2):167-76.

17. Mills DM, Wu CL, Williams DC, et al. High-fidelity simulation enhances pediatric residents' retention, knowledge, procedural proficiency, group resuscitation performance, and experience in pediatric resuscitation. Hosp Pediatr 2013;3(3):266-75.

18. Shah MI, Carey JM, Rapp SE, et al. Impact of High-Fidelity Pediatric Simulation on Paramedic Seizure Management. Prehosp Emerg Care 2016;20(4):499-507.

19. ChengA,BrownLL, DuffJP, etal.Improving cardiopulmonary resuscitation with a CPR feedback device and refresher simulations (CPR CARES Study): a randomized clinical trial. JAMA Pediatr 2015;169(2):137-44.

20. Nishiyama C, Iwami T, Murakami $Y$, et al. Effectiveness of simplified 15-min refresher BLS training program: a randomized controlled trial. Resuscitation 2015;90:56-60.

21. Mileder LP, Wegscheider T. Anaphylaxis management: a multimodal curriculum with a distinct focus on simulationbased training. Resuscitation 2014;85(10):e165-6.

22. Mileder LP, Kopaunik LS, Alberer M, etal.Simulation-based training and its effect on management of severe anaphylaxis-Are 4 Hs enough? Resuscitation 2016;98:e12-3.

23. Couto TB, FarhatSC, Geis GL, et al. High-fidelity simulation versus case-based discussion for teaching medical students in Brazil about pediatric emergencies. Clinics (Sao Paulo) 2015;70(6):393-9. 